\title{
Methodology for the Evaluation and Measurement of Therapeutic Progress
}

\author{
Didier Guillemot, ${ }^{1}$ Georges France, ${ }^{2}$ Pierre Fender ${ }^{3}$ and the Participants in Round Table No. 4, \\ Giens $X X^{*}$ \\ 1 CeRBEP, Institut Pasteur/Unité en Réseau U 657, INSERM, Paris, France \\ 2 Laboratoires Wyeth, Paris, France \\ 3 Assurance Maladie, Paris, France
}

\begin{abstract}
When a candidate drug is likely to become available to prescribers and healthcare policy makers, evaluation of therapeutic progress moves forward in two stages. First, the level of expected therapeutic progress must be established. This first stage requires the determination of therapeutic needs and the comparison of these against the results of the clinical studies that will form the basis of the marketing authorisation of the drug. This determination helps anticipate the therapeutic progress that is attributable to the approved use of the new drug. The second stage of the process, the evaluation of the actual therapeutic progress, involves therapeutic drug monitoring and bases itself on observation. Since such observational data are intended to challenge the initial hypotheses and uncertainties (in terms of benefits and risks), goals and methods must be laid out before the drug becomes available to the general public.
\end{abstract}

Keywords: therapeutic progress, epidemiology, evaluation

When penicillin $\mathrm{G}$ was introduced as a therapeutic drug for human use, the therapeutic progress was so clear that no one envisaged measuring it objectively. Moreover, this question apparently sparked no interest in either physicians or scientists at that time, because of the major innovation of the antibiotic and the tangible medical progress it represented. Clinical studies were sufficient for observing that its administration cured certain infections that, until that time, systematically killed the afflicted. Although the question of therapeutic progress went unaddressed, as important as it seemed, it was, nonetheless, important to demonstrate the efficacy of the drug. In addition, the implementation of an experimental protocol defining a random controlled study was not required for this molecule to receive marketing authorisation.

Since then, the context has changed. Today, no one would consider introducing a new therapeutic molecule onto the market without data on its efficacy, based on the results of clinical trials. In the same way, anticipating the therapeutic gain of a candidate drug and measuring real therapeutic progress, obtained through its use, have become indispensable.

\section{Do Not Confuse Therapeutic Progress with Pharmacological Innovation}

How do you define 'therapeutic progress'? The Littré dictionary ${ }^{[1]}$ can help give us a start:

- progress: any kind of increase in or advancement that is considered good;

- therapeutic: that which is related to the treatment of disease. From this, it would appear that therapeutic progress must be considered as a principle that refers, not to the inherent pharmacological characteristics of a given drug, but rather to what that therapeutic drug brings to the treatment of a given disease.

\footnotetext{
* For a list of participants, please see the end of the article.
} 
'Therapeutic progress' and 'pharmacological innovation' must not be confused. The former is relative to what will be possible to attain in terms of improving the benefits or decreasing the risks to patients or populations, while the latter refers to the biotechnological nature of the new pharmaceutical entity. 'Innovation' not only makes reference to progress in knowledge or the appearance of new concepts (e.g. Copernicus, Galileo, Einstein, Freud, etc.), but also to the perfection of a product or process that will potentially change the relationship between humans and their environment (e.g. Akrich). If we measure the essential nature of innovation when Fleming identified the antibacterial properties of penicillin $\mathrm{G}$, the most important characteristic of therapeutic progress can only be discerned by examining bacterial infections that penicillin $\mathrm{G}$ was able to cure or decrease the mortality rate of. Therapeutic progress speaks to the reality of the gain (therapeutic or preventive) that is linked to the use of a new drug. Human immunodeficiency virus (HIV) infection offers a good example, since the first antiretroviral agents (in existence for a little more than 10 years for the oldest among them) were significant therapeutic innovations. But, the main characteristics of the therapeutic progress relative to triple therapy were identified by research on patients treated by therapeutic combinations. There is a universal dimension to therapeutic innovation. It shapes the landscape of therapeutic progress in the real world, but only the real world. Therapeutic progress is contextual and depends on circumstances, its meaning dependent on therapeutic (or preventive) practices within the population concerned and at the moment the new drug becomes available to healthcare professionals and patients. This point is important since it supports the assertion that therapeutic progress forcibly evolves and, therefore, must be regularly re-evaluated and measured relative to other current therapeutic practices.

\section{The Three Steps of Measuring Therapeutic Progress}

The three steps of measuring therapeutic progress are characterising the needs, quantifying the expected therapeutic progress and measuring the observed therapeutic progress.

When addressing the measurement of therapeutic progress, both medical and public aspects have to be considered. ${ }^{[2]}$

The questions related to measuring therapeutic progress pose themselves at three distinct stages in the life of a drug. First, very early in the development of a candidate drug, there is the question of how much therapeutic progress is expected. This first consideration is essential, since it requires you to (i) determine the therapeutic needs before the drug is in use; (ii) confront what we know about the candidate drug in order to evaluate how it could respond to the identified needs; and (iii) generate an informed hypothesis about how much additional benefit the drug might offer, such as other issues the candidate drug could respond to. The two key terms here are: 'how much', because it is necessary to be able to obtain a predetermined estimate that is not exclusively qualitative; and 'hypothesis', because it is just as indispensable to know what level of credibility it is that you give this predetermination.

\subsection{First Step: Characterisation of Needs}

It is necessary to underscore that characterising needs is an exercise that requires precise and detailed descriptive epidemiological and pharmacoepidemiological data, such as reliable estimates of the number of patients concerned; the characterisation and distribution of the various stages of the disease; clinical evolution and prognosis, especially in terms of mortality, but also with regard to the disability of patients, which represents the future indication for the drug according to recent epidemiological changes; and the description of therapeutic practices and their influence on prognosis. The absence (or extreme rarity) of such data explains why the preliminary formalisation (qualitative or quantitative) of needs (from the clinical or epidemiological point of view) exists only rarely. Vaccines, in particular, are a good example of how the formalisation of needs can occur at a preliminary stage of development, because of the strong epidemiologic dimension involved in the therapeutic progress and because the anticipation of needs has already been initiated. ${ }^{[3]}$

Nonetheless, regardless of the rationalisation for needs analysis and the necessity for regular updates, there is a problem that cannot be resolved by experimental pharmacology, clinical research or epidemiology: the human factor. This factor can have an important influence on the result of the analysis as the subjectivity of various points of view and competetion come into play regarding the priority of certain choices. It is not a given that the points of view of the manufacturer of the candidate drug, healthcare providers, patients and the other professionals involved in the process will naturally and spontaneously converge in the needs analysis. However, the development methods of the candidate drug might all depend on this analysis, for example, focus it on a particular indication or on one population over another. One solution might be to organise, at a very early stage in the development of the drug, consultative committees that address this analysis and decide what development avenues should be focused on.

The epidemiological characterisation of the therapeutic need that a new molecule might respond to often remains imprecise because epidemiological studies and analyses relative to the tar- 
get population of the drug were not implemented early in the development process. It appears that the process of predicting the therapeutic progress of a candidate drug cannot wait for the drug to receive marketing authorisation, but must be initiated in the earliest stages of clinical development.

\subsection{Second Step: Quantification of the Expected Therapeutic Progress}

Another problem exists, which concerns the formalisation of the expected therapeutic progress. This problem relates to the nature of the data used in the creation of scenarios to illustrate expected therapeutic progress. For example, how much morbidity and mortality can be avoided by putting a new anti-cholesterol drug on the market? Although the data that are available on the drug at this stage result almost exclusively from clinical studies described in the marketing authorisation application, the response to this question requires that the hypothesis be credible and that uncertainties, such as the possible variability in future use of the product, be anticipated. These uncertainties include drug safety, especially with regard to the risk of serious or rare adverse effects that, by their natures, have not been identified during clinical development; the reality and state of optimising the clinical gains and, especially, the likely compliance in populations exposed to the drug; and the changing clinical context. Because the conditions under which clinical trials are carried out are only distant approximations of the real world in which the drug will be used, clinical trial results alone cannot lead to the credible formalisation of a hypothesis.

An ideal solution to this problem probably does not exist. A partial solution may be to undertake, at a very early stage in the development process of a candidate drug, studies that are aimed at estimating the therapeutic gain under conditions as close to the future reality as possible. However, in reality, this proposal is not without its weaknesses given that, as long as a candidate drug does not have marketing authorisation, it is impossible to imagine the experimental conditions of a clinical trial that might anticipate the future conditions of the real use of the drug. In that case, it is mathematical modelling, combined with reliable epidemiological and pharmacoepidemiological data, that must provide hypotheses regarding the future use of the drug.

At this stage, another methodological challenge must be overcome. The vast majority of clinical trials have been designed with the goal of concluding that the candidate drug is not inferior to the existing drug of reference. The results created by this approach (non-inferiority) do not allow the demonstration of the superiority of the candidate drug. They are inherently unsuitable for bringing therapeutic progress to light, except if a gain appears in areas other than those for which the efficacy had been evaluated during the clinical research phase. Nonetheless, there is no other option but to use these results.

The choice of criteria by which to measure expected therapeutic progress is especially important. First, it must be underscored that economic considerations have nothing to do with therapeutic considerations. Although the economic dimension remains an important element in public health policy making, it is a point of view that is independent of the therapeutic considerations. Regardless of the methods of measurement, survival must first of all be considered, followed by the risk of disease or handicap resulting from adverse events. Morbidity and mortality are, for this purpose, the strongest and most relevant indicators from the clinical and public health perspectives. After that, the therapeutic gain can be evaluated using quality-of-life criteria or the expected impact on healthcare management.

Regardless of the criteria selected, it is necessary to determine before the analysis of therapeutic progress the clinical and epidemiological relevance of the expected therapeutic gain. This needs to be determined, once again, very early in the development of the candidate drug and could rely on the committees that we evoked in the needs analysis.

Finally, all of this demonstrates the importance of addressing the question of expected therapeutic progress as early as possible in the developmental stages of drugs in the future. The formalisation of this hypothesis can be broken down into four parts:

1. quantification of expectations using relevant clinical and epidemiological indicators;

2. mutually agreed formalisation of specifications that describe, most notably, the data that are indispensable in revealing the therapeutic context in which the drug will be marketed (epidemiological and pharmacoepidemiological); creating a protocol for following patients not included in the clinical trials, whose data, in reality, will best represent the use of the drug in the target populations; and creating a system to analyse placebo groups in detail;

3. characterisation of target and additional populations before clinical trials are carried out;

4. quantified formalisation of the hypothesis, with a detailed description of methodology, especially when involving mathematical modelling, quantification of uncertainty and the definition of follow-up goals regarding future use of the drug, most notably drug safety and adaptation to populations, actual therapeutic gain and changing context. 


\subsection{Third Step: Measurement of the Therapeutic Progress}

Comparing expected therapeutic progress with the data used to measure actual therapeutic progress is essential because, without that comparison, it would be impossible to:

- reduce the uncertainty of the hypothesis and bring the new events concerning the risk and benefit of the drug to light;

- revise the hypothesis, especially to take into account the real modalities of usage and the temporal dynamics involved in the therapeutic context.

There is no single method for comparing the expected therapeutic process with the data, but rather a number of different possibilities that depend on the goals established at the outset. It is within this framework that the creation of cohorts, pragmatic trials and the use of databases can be contemplated, especially if they evolve by incorporating data from clinical observation and changing contexts.

\section{Conclusion}

Today, we too often have the unsatisfactory situation in which we must use the results of clinical trials to characterise the expected therapeutic progress for a new drug while knowing full well that extrapolating those results to cover the real usage of the molecule in the field is hazardous at best. For that reason, this Round Table has mainly focused its attention on showing that it is essential to anticipate, as early as possible in its development process, what a new drug can offer in terms of therapeutic progress.

\section{Participants}

J.-M. Alexandre (Afssaps, Saint-Denis), O. Amede-Manesme (Paris Biotech, Paris), J.-P. Bader (Vidal, Issy-les-Moulineaux), M. Bouhassira (Lilly France, Suresnes), B. Calles (LIR, Paris), A. Castaigne (Hôpital Henri Mondor, Paris), M. Chauvenet (Aremis, Neuilly), B. Diquet (CHU, Angers), I. Giri (Consultant, Verrières-le-Buisson), F. Ichou (Sanofi Aventis, Paris), P. Jolliet (Institut de Biologie, Nantes), J.-M. Joubert (Roche, Neuilly-surSeine), J.-P. Lehner (Sanofi Synthélabo, Gentilly), M. Lièvre (Faculté de Médecine, Lyon), H. Mathiex-Fortunet (Beaufour Ipsen Pharma, Paris), M. Marty (CNAMTS, Paris), F. Meyer (HAS, Paris), J. Micallef (Hôpital La Timone, Marseille), M. Pigeon (CNAMTS, Paris), B. Rouveix (Hôpital Cochin Saint-Vincent de Paul, Paris), F. Zannad (Hôpital Jeanne d'Arc, Dommartin les Toul).

\section{References}

1. Littré E. Dictionnaire de la Langue Française. Paris: Gallimard-Hachette, 1973

2. Déclaration de l'ISDB sur le progrès thérapeutique dans le domaine des médicaments. Paris: International Society of Drugs Bulletins, 2001

3. Vaccinations : actualités et perspectives. Expertise collective INSERM. Paris: INSERM, 1999; XIV: $349 \mathrm{p}$

Correspondence and offprints: Didier Guillemot, Centre Ressources Biostatistiques, Epidémiologie et Pharmaco-Epidémiologie appliquées au risque infectieux, 28 rue du Docteur Roux, 75724 Paris Cedex 13, France. E-mail: guillemo@pasteur.fr 